\title{
Experiences in Using MPI-IO on Top of GPFS for the IFS Weather Forecast Code
}

\author{
Nicholas K. Allsopp ${ }^{1}$, John F. Hague ${ }^{2}$, and Jean-Pierre Prost ${ }^{3}$ \\ 1 IBM Hursley Laboratories, Winchester, UK. \\ 2 IBM Bedfont Lakes, Feltham, Middlesex, UK. \\ 3 IBM T.J. Watson Research Center, Yorktown Heights, NY, USA.
}

\begin{abstract}
The Integrated Forecast System (IFS) code is a parallel MPI application running on multiple tasks, a specified number of which, during its execution, writes output to a single global file at the end of several output time intervals. It can therefore write output multiple times during a given run. With the appropriate choice of parallel writing routine the overhead of writing to disk can be effectively hidden from the computation. We shall show how this is possible with careful use of MPI-IO routines on top of the IBM General Parallel File System (GPFS).
\end{abstract}

\section{Introduction}

The Integrated Forecast System (IFS) [1,2] code is a parallel MPI [3,4] application running on multiple tasks, a specified number of which, during its execution, writes its outputs to a single global file at the end of several output time intervals. It can therefore write output multiple times during a given run. A serial application can often run out of I/O performance when using a disk system connected to a single processing node. It is already possible to stripe the data across the disks on one node. The next logical step is to stripe the data across multiple nodes thus balancing the I/O activity. The IFS application often needs to write data that is located on a disk which happens to be physically located on a different processor. Usually there is no flexible way to access data on any disk attached to any processor from within the application. The Network File System (NFS) [5] could provide such a solution but its performance is not adequate in many cases. This is particularly true for its write performance.

The IBM General Parallel File System (GPFS) [6] allows the flexibility of access to shared data across multiple nodes. Parallel applications such as IFS need access to disks that are spread across a number of nodes to achieve performance. GFPS allows multiple processors on several nodes to simultaneously access the same file using Posix file system calls. It utilizes the IBM SP [7] switch for fast access and increases the aggregate bandwidth by spreading reads and writes across multiple disks. To maximize the total throughput, it balances the load evenly across all disks. This means that it does not matter to which node the disks are attached. Several studies have been carried out by researchers $[8,9,10]$, aimed at characterizing the behaviour of I/O intensive parallel applications and 
Table 1. Possible MPI-IO functions that could be used for writing to a single file

\begin{tabular}{lll}
\hline Function & Synchronization & Positioning of File pointer \\
\hline MPI_FILE_WRITE_ORDERED & Blocking Collective & Shared \\
MPI_FILE_IWRITE_SHARED & Nonblocking Independent Shared \\
MPI_FILE_WRITE_ALL & Blocking Collective & Individual \\
MPI_FILE_IWRITE & Nonblocking Independent Individual \\
\hline
\end{tabular}

at observing the performance gain which can be obtained by restructuring the application code. What we will show in this paper is that with the appropriate choice of parallel writing routine the overhead of writing to disk can be effectively hidden from the computation. This is particularly important when considering an application such as IFS which not only has a lot of calculation but outputs large amounts of data at regular intervals.

\section{The IFS Application Code}

IFS is the Integrated Forecast System program [1] developed by ECMWF (European Centre for Medium-Range Weather Forecasts) for producing a global 10 day weather forecast. The code is based on a spectral model, with specific development to make it suitable for a distributed memory parallel architecture. The forecast data can be written to a global file system (or database) as frequently as required. Typically, this would be after every 3 hours of prediction, although in this case we chose to output the data for every 15 minutes of prediction to exercise the GPFS system as much as possible.

Within the file writing routine of the IFS code we envisage a scenario where every 30 seconds, each of 1000 MPI tasks outputs 500 messages of $2 \mathrm{~KB}$ each. This causes $1 \mathrm{~GB}$ to be written into a single file. The messages have to be fully interleaved in the output file. In the IFS code they are sent by each MPI task either to one master task which writes them out sequentially, or to several (user determined) master tasks which write them out using MPI-IO calls.

\section{Choices of MPI-IO Writing Functions}

Table 1 shows four functions that could easily be used within the IFS code. For the case where the file pointer is not shared, the user needs to specify the information about the structure of the file to the MPI tasks that are performing the file transfer through the setting of appropriate file views. Of these four MPI-IO functions, MPI_FILE_IWRITE_SHARED appears the most appropriate to be used within the IFS application. This is because it requires the least code modification and the file block sizes might not be the same on each MPI task (therefore using an individual file pointer routine would require a call to MPI_FILE_SET_VIEW function at the beginning of each output phase as well 


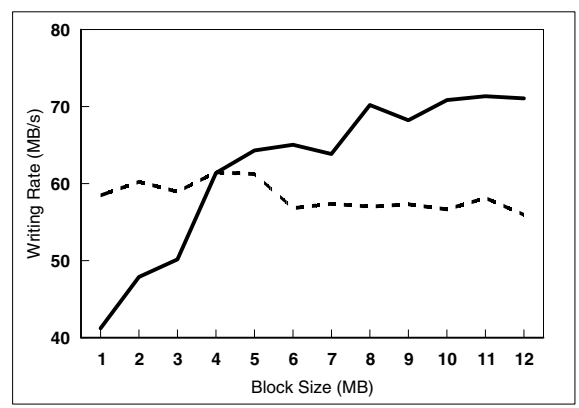

Fig. 1. Speed for writing a single $1 \mathrm{~GB}$ file to disk from 8 nodes using both a single master task (dotted line) and 8 MPI-IO master tasks (solid line)

as additional communication between MPI tasks). As seen in Figure 1, writing with a single master task gives a maximum bandwidth of around $60 \mathrm{MB} / \mathrm{s}$. When using MPI-IO and 8 master tasks the aggregate write bandwidth becomes higher for block sizes over $4 \mathrm{MB}$ to reach $70 \mathrm{MB} / \mathrm{s}$ for block sizes over $10 \mathrm{MB}$. For larger block sizes, the MPI-IO overhead is compensated by multiple GPFS clients writing in parallel to the file.

Although MPI-IO does not appear to be much faster than a serial write, the important thing here is that, when using MPI-IO, all tasks can participate. It should be noted that although 70MB was perfectly adequate, more comprehensive GPFS configurations are capable of achieving 200 to $300 \mathrm{MB} / \mathrm{sec}$.

\section{IFS Test Case}

While benchmark programs (like the previous example) can give an indication of the performance of new systems, their extrapolation to real-world application performance is not always straightforward. In this section, we use the IFS code to test the application performance of our GPFS file system. The test case used (T159) is a global forecast spectral model with 60 level, 320 longitudes and 160 latitudes. ECMWF runs much higher resolution models for the actual forecast so we are here concentrating on the I/O aspects of the runtime. This model outputs $44 \mathrm{MB}$ of data per calculation step and there were 24 steps per run.

The IFS has the capability to have either a single master task writing all the data to the single file, meaning that all the other tasks must communicate their portion of the output file to the master task, or have a number of parallel writing tasks which use MPI-IO to write to a single file. The number of parallel writing tasks can be the same as or less than the total number of tasks used during the computation. If the number of writing tasks is less than the total number of tasks then there is some inter-task communication involved in order to package the output data ready for file writing. 
Table 2. Execution times on the SP test platform of the IFS code using MPI_FILE_IWRITE_SHARED for the parallel write command. The 'dummy' numbers are for IFS codes using dummy parallel write commands. All times are in seconds

\begin{tabular}{|c|c|c|c|c|c|c|c|c|c|}
\hline \multirow{2}{*}{\multicolumn{2}{|c|}{$\begin{array}{cc}\text { \# Tasks } & 1 \text { Task } \\
\text { Total writing }\end{array}$}} & \multicolumn{2}{|c|}{2 Tasks } & \multicolumn{2}{|c|}{4 Tasks } & \multicolumn{2}{|c|}{8 Tasks } & \multicolumn{2}{|c|}{16 Tasks } \\
\hline & & writing & 'dummy' & writing & 'dummy' & writing & 'dummy' & writin & dummy' \\
\hline 4 & 1584 & 1577 & 1556 & 1540 & 1540 & - & - & - & - \\
\hline 8 & 857 & 816 & 816 & 801 & 800 & 777 & 780 & - & - \\
\hline 16 & 564 & 561 & 519 & 527 & 495 & 539 & 499 & 558 & 490 \\
\hline
\end{tabular}

The values shown in table 2 were obtained from the IFS test case running on the SP test platform. For comparsion this test was repeated on the most upto date SP2 platform consisting of a single Night Hawk 2 (NH2) node as shown in table 3 . Table 2 shows the difference in execution time when using a single master task to write the file compared to using multiple tasks writing to a parallel shared file with MPI-IO. Although it is obvious that the parallel file writing routine outperforms the single master task approach, there is a trade-off between the communication overhead induced by passing the data to a single master task and the MPI-IO overhead of accessing a shared file pointer compensated by the parallel access to the file by several master tasks. This can be seen from the results of using a total number of 16 tasks as the shortest execution time was obtained when using 4 writing tasks. The results of table 2 'dummy' were

Table 3. Execution time on latest SP2 platform using a 16 CPU NH2 node. All times in seconds

\begin{tabular}{cccccc}
\hline \# Tasks & 1 Task & 2 Tasks & 4 Tasks & Tasks & Tasks \\
writing & writing & writing & writing & writing \\
\hline 4 & 908 & 868 & 840 & - & - \\
8 & 469 & 451 & 450 & 448 & - \\
16 & 275 & 279 & 266 & 266 & 264 \\
\hline
\end{tabular}

obtained by replacing the MPI-IO file write routines by dummy routines thus removing any file writing whilst retaining all of the file packing algorithms. There is a larger difference shown when using larger numbers of tasks but this is expected because the block size per write is small causing a drop in write performance, as shown in Fig. 1. This shows that actual overhead of the parallel file writing, as performed by MPI-IO on top of GPFS is very small and is not causing a bottleneck for the calculation stage of the IFS test case. 


\section{Conclusion and Future Work}

The results above show that parallel file writing is faster than using a single master writing task. There is a trade-off between the communication overhead induced by passing the data to a single master task and the MPI-IO overhead of accessing a shared file pointer compensated by the parallel access to the file by several master tasks. For this reason, careful consideration is required when choosing the number of master tasks. As can be seen in table 2 , it is not straightforward to determine the optimal number of master tasks when using MPI-IO. The main reason for finding the optimal method of file writing is to effectively hide the I/O overhead such that calculation dominates the overall execution time. This is particularly important when considering an application such as IFS which not only has a lot of calculation but outputs large amounts of data at regular intervals. We have shown that this is possible when using an appropriate number of MPI-IO writing tasks compared to using the single master task approach.

In the future, we are planning to run the IFS code on a larger test case and with more tasks, since the typical IFS 10 day forecast model currently runs with a grid size of about 1000 longitudes by 500 latitudes.

\section{Acknowledgment}

The authors would like to thank ECMWF for supporting this work.

\section{References}

1. Barros, S. R. M., Dent D., Isaksen, I., Robinson, G., Mozdzynski, G., Wollenwever, F., The IFS model: a parallel production weather code. Parallel Computing, 21 No.10 (1995) 1621-1638.

2. The IFS Model Overview and Parallelisation Strategies, Proceedings of the 6th ECMWF workshop, 1994. ISBN 981-01-2211-4.

3. Message Passing Interface Forum, MPI-2: Extensions to the Message-Passing Interface, July 1997. Http://www.mpi-forum.org/docs/docs.html.

4. Gropp, W., Lusk, E., and Thakur, R., Using MPI-2: Advanced Features of the Message-Passing Interface. MIT Press, Cambridge, MA, 1999.

5. The GPFS parallel file system: http://www.almaden.ibm.com/cs/gpfsspsort.html.

6. The Network File System (NFS): http://www.nfsv4.org/

7. The IBM SP system: http://www.rs6000.ibm.com/hardware/largescale/SP/index.html

8. Kotz, D. and Nieuwejaar, N., Dynamic file-access characteristics of a production parallel scientific workload. In Proceedings of Supercomputing '94, 640-649, (1994). IEEE Computer Society Press.

9. Nieuwejaar, N., Kotz, D., Purakayastha, A., Schlatter Ellis, C., and Best, M., Fileaccess characteristics of parallel scientific workloads. IEEE Transactions on Parallel and Distributed Systems, 7(10) (1996) 1075-1089. 
10. Smirni, E., and Reed, D. A., Lessons from characterizing the input/output behavior of parallel scientific applications. Performance Evaluation: An International Journal, 33(1) (1998) 27-44. 